\title{
Quand certains flavivirus remettent en cause nos certitudes
}

\section{When some Flaviviruses are throwing our certainties}

\section{Chastel}

Reçu le 24 mai 2012 ; accepté le 26 juin 2012

(C) Société de pathologie exotique et Springer-Verlag France 2012

Résumé Au cours des deux dernières décennies, on a assisté à l'émergence brutale d'un certain nombre de flavivirus transmis par des moustiques, principalement d'origine africaine, envahissant des régions géographiques où ils n'avaient jamais été actifs. Ce fut le cas pour le virus de l'encéphalite japonaise (JEV) qui, à partir de 1995, a gagné le Nord-Est de l'Australie et, surtout, du virus West Nile (WNV) qui a colonisé tout le continent américain à partir de 1999. Puis, dès 2001, le virus Usutu (USUV) a infecté une grande partie de l'Europe occidentale et le virus Zika (ZIKV) a provoqué une sérieuse épidémie en Micronésie (2007). Enfin, en 2010, le virus Tembusu (TMUV) a ravagé des élevages de canards en Chine, tandis que le virus Bagaza, après s'être manifesté au Kerala, en Inde, par des cas d'encéphalites humaines, a infecté le Sud de l'Espagne. Des pouvoirs pathogènes nouveaux ont été observés chez l'Homme et chez l'animal, en même temps que des voies inhabituelles de contamination étaient décrites et que ces virus infectaient des hôtes vertébrés nouveaux et de nouvelles espèces de moustiques. Les causes de cette évolution inattendue restent obscures.

Mots clés flavivirus transmis par des moustiques · Invasion de nouvelles régions - Pouvoirs pathogènes nouveaux $\cdot$ Voies inhabituelles de contamination

\begin{abstract}
During the past two decades, a number of mosquito-borne flaviviruses, mainly of African origin, have invaded new geographical areas where they have never been active. This was the case for the Japanese encephalitis virus which reached the northeastern part of Australia (1995) and, above all, for the West Nile virus which, since 1999, entirely colonized the American continent. Then, the Usutu virus invaded a large part of the Western Europe (2001) while the Zika virus caused a large epidemic in an island of Micronesia (2007). Finally, in 2010, the Tembusu virus devastated
\end{abstract}

C. Chastel $(\bowtie)$

3, rue Rouget-de-l'Isle, F-29200 Brest, France

e-mail : chastelc@aol.com many duck farms in China while the Bagaza virus, after having provoked human encephalitis in India, reached the southern part of Spain. In the affected areas, new pathogenic outcomes were observed in humans and animals while new vertebrate hosts and mosquito species were infected. Moreover, unusual ways of contamination were described. The origins of this unprecedented evolution remain to be clarified.

Keywords Mosquito-borne flaviviruses · Invasion of new areas - New pathogenic outcomes · Unusual ways of contamination

\section{Introduction}

En moins de 20 ans, certains flavivirus transmis par des moustiques ont manifesté des capacités évolutives et invasives inattendues. Ces virus, transmis par des moustiques, échappés de leurs foyers naturels d'infection tropicaux ou subtropicaux, ont envahi de nouvelles régions, voire des continents entiers. Ils ont aussi acquis un pouvoir pathogène accru pour l'Homme et pour les animaux sauvages ou domestiques. Ils ont infecté de nouveaux hôtes vertébrés et de nouvelles espèces de moustiques, tout en adoptant des modes de transmission inhabituels.

\section{Genre flavivirus}

Il rassemble des virus enveloppés, sphéroïdaux, de 40 à $60 \mathrm{~nm}$ de diamètre. Leur génome, inclus dans une capside icosaédrique, est constitué d'un seul brin d'ARN, de polarité positive. Les 9600 à 11000 nucléotides qui le composent codent sept protéines non structurales et trois protéines de structure. Plus de 70 flavivirus ont été décrits. Ce sont pour la plupart des agents de zoonoses.

Ces virus provoquent chez l'Homme des infections inapparentes, des états fébriles indifférenciés, des hépatites, des fièvres hémorragiques et des atteintes du système nerveux. Leur analyse phylogénique a permis de définir quatre 
groupes génomiques et écologiques : deux sont transmis par des moustiques, un est transmis par des tiques et le dernier rassemble des virus qui n'ont pas de vecteur connu [20,21].

\section{Échappées récentes de flavivirus hors de leurs aires géographiques habituelles}

En 1995, le virus de l'encéphalite japonaise (JEV) a quitté le Sud de la Papouasie-Nouvelle-Guinée, gagné les îles du détroit de Torres, puis infecté le Nord-Est de l'Australie, probablement transporté par un oiseau infecté ou par des moustiques poussés par le vent [34]. Il y a infecté des espèces locales de moustiques, des porcs domestiques et quelques personnes [34,49,50].

En 1995, le virus West Nile (WNV), échappé du MoyenOrient, a progressivement envahi le Nouveau Monde, y infectant de nombreuses espèces d'oiseaux, de mammifères terrestres et marins, de crocodiliens et de moustiques, tout en provoquant des encéphalites, souvent mortelles, chez l'Homme [11].

Le virus Usutu (USUV), un flavivirus d'origine africaine, transmis par des moustiques, s'est solidement implanté dans l'avifaune sauvage de l'Europe centrale à partir de 2001 $[52,53]$. L'intrusion a débuté dans la région de Vienne, où il a provoqué d'importantes mortalités chez le merle noir (Turdus merula) et chez des chouettes lapones (Strix nebulosa) du zoo de Schönbrunn. Puis, $200 \mathrm{~km}$ plus à l'ouest, des hirondelles rustiques (Hirundo rustica) furent trouvées mortes. Le virus isolé de ces oiseaux, d'abord confondu avec le WNV, était en fait l'USUV, un virus jamais rencontré en Europe. Le séquençage complet des souches autrichiennes a montré qu'elles étaient très proches de la souche sudafricaine de référence (SAAR-1776) [2]. En fait, ce virus était implanté en Autriche dès 2000 [52]. C'était la première fois qu'il manifestait un pouvoir pathogène élevé pour des animaux, mais l'infection paraissait géographiquement localisée [53].

Tout a changé, en 2007, lorsque les autorités sanitaires italiennes, à la suite de l'épidémie de Chikungunya, ont entrepris une vaste enquête entomologique en ÉmilieJulienne. À partir de lots de Culex pipiens et d'Aedes albopictus, ils ont détecté 27 fois le WNV, 56 fois l'USUV, les deux virus infectant simultanément huit lots $[7,46]$. De plus, l'USUV avait infecté dans le Nord de l'Italie des merles noirs et des chouettes en captivité, les souches virales étant génétiquement identiques à celles de l'Europe centrale [35]. Enfin, les deux premiers cas humains d'infection neuroinvasive par ce virus furent décrits en Italie $[10,18,41]$. Il s'agissait de femmes immunodéprimées, polytransfusées, présentant des pathologies associées lourdes, cancéreuses ou hématologiques.

Mais la diffusion géographique de l'USUV en Europe occidentale était, en réalité, plus large. Il avait infecté des oiseaux sauvages ou en captivité, des poulets sentinelles et des moustiques, en République tchèque, en Pologne et en Suisse [26], au Royaume-Uni [5], en Hongrie [3], en Espagne [6,51] et en Allemagne [28].

Le virus Zika (ZIKV), isolé en 1947 en Ouganda d'un singe Macacca mulatta, est un flavivirus transmis par des moustiques dont la répartition géographique est très large : du Sénégal aux Philippines [37,38]. Toutefois, on savait peu de chose de son pouvoir pathogène chez l'Homme : essentiellement des états fébriles indifférenciés, sans gravité [37].

Puis, brusquement, d'avril à juin 2007, le ZIKV s'est manifesté par une violente épidémie dans l'île de Yap, en Micronésie [16,32]. Les malades avaient de la fièvre, une éruption maculopapuleuse, de la conjonctivite et des arthralgies. L'ARN obtenu du sang des malades était celui du ZIKV lequel n'avait jamais été rencontré dans cette région. La recherche des IgM spécifiques a montré que $71 \%$ de la population avait été infectée. Aucun décès ne fut à déplorer. Aedes hensilli était le moustique le plus abondant sur l'île, mais les tentatives pour en isoler le virus furent infructueuses [16]. Le ZIKV avait frappé massivement une population immunologiquement vierge.

Sur le plan moléculaire, la souche prototype de ZIKV (MR 766) avait été entièrement séquencée [31]. Celle de Yap, quoique génétiquement proche, en était cependant distincte, ainsi que des souches isolées au Sénégal [31,32].

Le virus Tembusu (TMUV) est un flavivirus, transmis par des moustiques, isolé en 1955, de Culex tritaeniorhynchus, en Malaisie [29]. Il fut ensuite isolé chez de très nombreuses espèces de moustiques en Malaisie et au Sarawak [42]. On ne lui connaissait pas de pouvoir pathogène chez l'Homme ou chez l'animal, mais on l'avait isolé de poulets sentinelles, et $70 \%$ des poulets domestiques avaient des anticorps contre ce virus au Sarawak [42].

Puis, brutalement, en 2010, le TMUV émergea en Chine sous la forme d'une épizootie dévastatrice dans des fermes où l'on élevait des canards : $90 \%$ des oiseaux furent atteints avec une mortalité de 5 à $30 \%$. La production d'œufs chuta de façon drastique [8]. Un flavivirus fut isolé des ovaires des canes malades, et il présentait une identité nucléotidique de 87 à $91 \%$ avec le TMUV; il fut classé comme un génotype particulier de ce virus [8]. Mais, lorsque l'on séquença ce nouveau génotype, on s'aperçut qu'il s'agissait d'un flavivirus nouveau, le virus Baiyandian (BYDV) [45]. Le BYDV est également apparenté au virus Sitiawan (SV) isolé de cas d'encéphalite chez des poulets en Malaisie [30] et au virus Bagaza (BAGV) [45].

Le BAGV est un flavivirus isolé en 1966, en RCA, d'un lot de Culex $s p$., puis de très nombreuses espèces de moustiques au Cameroun, au Sénégal et en Mauritanie $[15,29,48]$. Lorsque le BAGV fut entièrement séquencé, des similitudes avec le WNV apparurent [31]. Mais, c'est seulement en 1996 que le BAGV a révélé sa dangerosité potentielle chez 
l'Homme, lorsqu'il fut isolé de $C x$. tritaeniorhynchus lors d'une épidémie d'encéphalite au Kerala en Inde : $15 \%$ des malades avaient des anticorps NT contre le BAGV [4]. Ce nouvel isolat partageait une identité nucléotidique de 94,8\% avec la souche africaine Dakar B 209. De plus, à l'intérieur du groupe Ntaya des flavivirus, il apparaissait étroitement apparenté au virus Israel Turkey meningo encephalitis (ITMV) et de façon plus distante avec le TMUV [4,31].

Enfin, en septembre 2010, le BAGV fut identifié près de Cadix, dans le Sud de l'Espagne où il provoquait de fortes mortalités chez la Perdrix rouge (Alectoris rufa) et le Faisan de Colchide (Phasianus colchicus) [1]. Des tissus des oiseaux morts, on isola le virus et son séquençage complet montra une plus grande similitude génétique avec Dakar B 209 (94,1 \%) qu'avec la souche indienne 96363 (92,8 \%). De plus, la souche espagnole était génétiquement très proche de l'ITMV [1].

Les quatre virus de la dengue (DENV 1-4) sont, parmi les flavivirus transmis par des moustiques, ceux dont la diffusion mondiale a été la plus forte au cours des dernières années. La dengue est maintenant la première arbovirose menaçant l'humanité. On estime que 2,5 milliards d'individus vivent dans des zones d'endémie et que 50 à 100 millions de cas surviennent chaque année, provoquant 500000 hospitalisations [24] et environ 20000 décès [44].

Comme conséquence directe de l'installation du moustique Ae. albopictus dans plusieurs pays d'Europe, ceux-ci sont exposés à l'apparition de petits foyers de transmission, voire à des épidémies. C'est notamment le cas pour l'Italie [43], pour la France [33] et la Croatie [19]. Dans chacun de ces deux derniers pays, deux cas autochtones sont survenus en septembre 2010. C'est la première fois qu'une transmission autochtone de la dengue est décelée en Europe depuis l'épidémie historique de la Grèce en 1927-1928 [12].

\section{Caractères de ces invasions}

Elles ont un caractère soudain et imprévisible, plaçant les autorités sanitaires dans l'embarras, car le virus n'est pas connu localement, son identification virologique est plus ou moins délicate et les réactifs adéquats ne sont pas disponibles. Lors de l'arrivée du WNV à New York, en 1999, il a d'abord été confondu avec le virus de l'encéphalite de SaintLouis, puis avec le virus Kunjin, avant d'être correctement identifié [11]. De même, quand l'USUV a émergé en Autriche, il a été confondu avec le WNV [52]. Mais ces intrusions ont d'autres particularités.

\section{Infection de nouveaux hôtes vertébrés et invertébrés}

Pour l'essentiel, ce sont des oiseaux, sauvages ou en captivité, qui ont été la cible de l'USUV [3,35,52,53]. Les vec- teurs étaient $C x$. pipiens et Ae. albopictus [6,7,28,46,51]. Pour le ZIKV, le vecteur en Micronésie a pu être Ae. hensilli [16].

Les hôtes vertébrés du TMUV étaient des poulets domestiques au Sarawak [42] et des canards d'élevage en Chine $[8,45]$. Au Sarawak, le vecteur est très vraisemblablement Cx. tritaeniorhynchus [42]. Enfin, le BAGV a apparemment provoqué des encéphalites chez l'Homme en Inde [4] et de fortes mortalités chez des oiseaux sauvages dans le Sud de l'Espagne [1]. Au Kerala, son vecteur est très probablement Cx. tritaeniorhynchus [4].

\section{Un pouvoir pathogène nouveau et des voies de contamination inhabituelles}

Tout comme le WNV en Amérique du Nord [25], la plupart de ces flavivirus ont provoqué chez l'Homme des infections neuro-invasives, jusqu'à présent non observées. Ce fut le cas pour l'USUV en Italie $[10,18,41]$ et le BAGV en Inde [4]. Plus surprenant, on a observé une fièvre hémorragique mortelle chez un Américain de 59 ans, en Floride, due au WNV [39] et des infections persistantes avec présence de ce même virus dans les urines [36].

Presque tous ces virus, y compris les quatre DENV, ont pu être transmis de façon inhabituelle : transfusion sanguine, transplantation d'organe, piqûre par une aiguille souillée $[9,14,23,27,40,54]$ ou pendant la période périnatale [47]. Enfin, une infection à ZIKV, probablement transmise par voie sexuelle, a été rapportée [17].

\section{Pourquoi de telles échappées invasives ?}

Elles sont difficiles à expliquer. On peut toutefois invoquer certains facteurs.

\section{Facteurs généraux}

Ce sont les mêmes que ceux qui ont été retenus dans les autres émergences virales récentes [11] :

- l'augmentation du trafic aérien international et la rapidité des transports, faisant que de plus en plus de voyageurs virémiques, infectés dans des régions d'endémie, peuvent introduire des flavivirus dans des régions non endémiques $[13,14]$;

- l'importance du volume des échanges commerciaux internationaux et la diversité des produits transportés, ce qui permet la diffusion mondiale de certains vecteurs comme Ae. albopictus ou l'introduction d'animaux exotiques infectés, importés légalement ou illégalement ;

- l'accroissement continu de la population mondiale, avec ses conséquences humaines et environnementales 
désastreuses : urbanisation anarchique, déforestation, extension de la riziculture, etc.

\section{Facteurs favorisants}

Il s'agit surtout de facteurs climatiques, tels que le réchauffement planétaire per se et les phénomènes climatiques du type El Niño/La Niña ou l'oscillation nord-atlantique (NAO). Ils ont une influence directe ou indirecte, positive ou négative, sur la répartition spatiale et l'activité des vecteurs, le niveau de la réplication virale chez ces derniers et l'abondance ou la disparition passagère d'hôtes vertébrés réceptifs.

\section{Facteurs génétiques}

Les flavivirus comme tous les virus à $\mathrm{ARN}$ sont génétiquement instables. Ils présentent des mutations [22] et peuvent éventuellement se recombiner [20,21]. Si les souches de USUV ayant diffusé en Europe sont restées génétiquement proches de la souche de référence africaine, la souche de ZIKV ayant émergé en Micronésie est distincte des souches isolées au Sénégal [31,32]. Le TMUV qui a envahi les élevages de canards en Chine fut d'abord classé comme un nouveau génotype de ce virus [8], puis finalement identifié comme un nouveau flavivirus, le BYDV [45]. Parmi les souches récemment émergées du BAGV, celle qui a été isolée en Inde s'est montrée génétiquement plus distante de la souche sénégalaise B 209 que celle isolée dans le Sud de l'Espagne $[1,4,31]$.

\section{Conclusion}

Nous venons d'assister, au cours des 20 dernières années, à l'émergence répétée de flavivirus transmis par des moustiques ayant quitté les aires géographiques où ils semblaient cantonnés, pour en gagner de nouvelles.

À l'exemple du WNV en Amérique, certains d'entre eux ont colonisé de vastes parties d'un continent, comme l'a fait l'USUV en Europe occidentale.

Dans les régions où ces virus ont pénétré, ils ont manifesté un pouvoir pathogène jusque-là inconnu pour l'Homme ou l'animal. Ils ont infecté des moustiques et des hôtes vertébrés nouveaux, principalement des oiseaux. Ils ont parfois provoqué des pertes économiques considérables, comme lorsque le BYDV a ravagé des élevages de canards en Chine.

Devant ces événements inattendus, les autorités sanitaires ont souvent été désemparées, les virus responsables apparaissant « nouveaux » et les techniques virologiques adéquates n'étant pas toujours disponibles.
On s'explique mal cette évolution, même si certains facteurs environnementaux, socio-économiques, climatiques ou génétiques peuvent avoir joué un rôle.

Conflit d'intérêt : l'auteur déclare ne pas avoir de conflit d'intérêt.

\section{Références}

1. Agüero M, Fernández-Pinero J, Buitrago D, et al (2011) Bagaza virus in partridges and pheasants, Spain, 2010. Emerg Inf Dis 17 (8):1498-501

2. Bakonyi T, Gould EA, Kolodziejek J, et al (2004) Complete genome analysis and molecular characterization of Usutu virus that emerged in Austria in 2001: comparison with the South African strain SAAR-1776 and other flaviviruses. Virology 328 (2):301-10

3. Bakonyi T, Erdélyi K, Ursu K, et al (2007) Emergence of Usutu virus in Hungary. J Clin Microbiol 49(12):3870-4. Epub 2007 Oct 3

4. Bondre VP, Sapkal GN, Yergolkar PN, et al (2009) Genetic characterization of Bagaza virus (BAGV) isolated in India and evidence of anti-BAGV antibodies in sera collected from encephalitis patients. J Gen Virol 90(Pt 11):2644-9

5. Buckley A, Dawson A, Gould EA (2006) Detection of seroconversion to West Nile virus, Usutu virus and Sindbis virus in UK sentinel chickens. Virol J 3:71

6. Busquets N, Alba A, Allepuz A, et al (2008) Usutu virus sequences in Culex pipiens (Diptera: Culicidae), Spain. Emerg Infect Dis 14(5):861-3

7. Calzolari M, Gaibani P, Bellini R, et al (2010) Mosquito, bird and human surveillance of West Nile and Usutu viruses in EmiliaRomagna region (Italy) in 2010. PLoS One 7(5):e38058. Epub 2012 May 30

8. Cao Z, Zhang C, Liu Y, et al (2011) Tembusu virus in ducks, China. Emerg Infect Dis 17(10):1873-5

9. Capobianchi MR, Sambri V, Castilletti C, et al (2010) Retrospective screening of solid organ donors in Italy, 2009, reveals unpredicted circulation of West Nile virus. Euro Surveill 15(34). pii: 19648

10. Cavrini F, Gaibani P, Longo G, et al (2009) Usutu virus infection in a patient who underwent orthotropic liver transplantation, Italy, August-September 2009. Euro Surveill 14(50). pii: 19448

11. Chastel C (2007) Les virus bougent : périls planétaires. Bull Acad Natl Med 191(8):1563-77

12. Chastel C (2009) En 1927-1928, la dengue s'abattait sur la Grèce : les enseignements d'une épidémie. Bull Acad Natl Med 193(2):485-93

13. Chastel C (2011) Infections inapparentes chez l'Homme : un cheval de Troie pour l'introduction et la diffusion des arbovirus transmis par des moustiques dans les régions non endémiques. Bull Soc Pathol Exot 104(3):213-9

14. Chastel C (2012) Eventual role of asymptomatic cases of dengue for the introduction and spread of dengue viruses in non-endemic regions. Front Physiol 3:70. Epub 2012 Mar 30

15. Diallo M, Nabeth $\mathrm{P}, \mathrm{Ba} \mathrm{K}$, et al (2005) Mosquito vectors of the 1998-1999 outbreak of Rift Valley Fever and other arboviruses (Bagaza, Sanar, Wesselsbron and West Nile) in Mauritania and Senegal. Med Vet Entomol 19(2):119-26

16. Duffy MR, Chen TH, Hancock WT, et al (2009) Zika virus outbreak on Yap Island, Federated States of Micronesia. New Engl J Med 360(24):2536-43 
17. Foy BD, Kobylinski KC, Chilson Foy JL, et al (2011) Probable non-vector-borne transmission of Zika virus, Colorado, USA. Emerg Infect Dis 17(5):880-2

18. Gaibani P, Pierro AM, Cavrini F, et al (2010) False-positive transcription-mediated amplification assay detection of West Nile virus in blood from a patient with viremia caused by an Usutu virus infection. J Clin Microbiol 48(9):3338-9. Epub 2010 Jun 30

19. Gjenero-Margan I, Aleraj B, Krajcar D, et al (2011) Autochthonous dengue fever in Croatia, August-September 2010. Euro Surveill 16(9). pii: 19805

20. Gould EA, de Lamballerie X, Zanotto PM, Holmes EC (2003) Origins, evolution, and vector/host coadaptations within the genus flavivirus. Adv Virus Res 59:277-314

21. Gould EA, Solomon T (2008) Pathogenic flaviviruses. Lancet 371(9611):500-9

22. Grard G, Moureau G, Charrel RN, et al (2010) Genomics and evolution of Aedes-borne flaviviruses. J Gen Virol 91(Pt 1):8794. Epub 2009 Sep 9

23. Grazzini G, Liumbruno GM, Pupella S, et al (2008) West Nile virus in Italy: a further threat to blood safety, a further challenge to the blood system. Blood Transfus 6(4):235-7

24. Halstead SB (2007) Dengue. Lancet 370(9599):1644-52

25. Hayes EB, Sejvar JJ, Zaki SR, et al (2005) Virology, pathology, and clinical manifestations of West Nile virus disease. Emerg Infect Dis 11(8):1174-9

26. Hubálek Z (2008) Mosquito-borne viruses in Europe. Parasitol Res 103(Suppl 1):S29-S43

27. Iwamoto M, Jernigan DB, Guasch A, et al (2003) Transmission of West Nile virus from an organ donor to four transplant recipients. New Engl J Med 348(22):2196-203

28. Jöst A, Bialouski A, Maus D, et al (2011) Isolation of Usutu virus in Germany. Am J Trop Med Hyg 85(3):551-3

29. Karabatsos N (1985) International Catalogue of Arboviruses including certain other viruses of vertebrates, $3^{\text {rd }}$ ed. American Society of Tropical Medicine and Hygiene, San Antonio

30. Kono Y, Tsukamoto K, Abd Hamid M, et al (2000) Encephalitis and retarded growth of chicks caused by Sitiawan virus, a new isolate belonging to the genus flavivirus. Am J Trop Med Hyg 63(1-2):94-101

31. Kuno G, Chang GJ (2007) Full-length sequencing and genomic characterization of Bagaza, Kedougou, and Zika viruses. Arch Virol 152(4):687-96. Epub 2007 Jan 3

32. Lanciotti RS, Kosoy OL, Laven JJ, et al (2008) Genetic and serologic properties of Zika virus associated with an epidemic, Yap State, Micronesia, 2007. Emerg Infect Dis 14(8):1232-9

33. La Ruche G, Souarès Y, Armengaud A, et al (2010) First two autochthonous dengue virus infections in metropolitan France, September 2010. Euro Surveill 15(39):19676

34. Mackenzie JS, Johansen CA, Ritchie SA, et al (2002) Japanese encephalitis as an emerging virus: the emergence and spread of Japanese encephalitis virus in Australasia. Curr Top Microbiol Immunol 267:49-73

35. Manarolla G, Bakonyi T, Gallazzi D, et al (2010) Usutu virus in wild birds in northern Italy. Vet Microbiol 141(1-2):159-63. Epub 2009 Aug 8

36. Murray K, Walker C, Herrington E, et al (2010) Persistent infection with West Nile virus years after initial infection. J Infect Dis 201(1):2-4
37. Olson JG, Ksiazek TG, Suhandiman, Triwibowo (1981) Zika virus, a cause of fever in Central Java, Indonesia. Trans $\mathrm{R}$ Soc Trop Med Hyg 75(3):389-93

38. Olson JG, Ksiazek TG, Gubler DJ, et al (1983) A survey for arboviral antibodies in sera of humans and animals in Lombok, Republic of Indonesia. Ann Trop Med Parasitol 77(2):131-7

39. Paddock CD, Nicholson WL, Bhatnagar J, et al (2006) Fatal hemorrhagic fever caused by West Nile virus in the United States. Clin Infect Dis 42(11):1527-35. Epub 2006 Apr 27

40. Pealer LN, Marfin AA, Petersen LR, et al (2003) Transmission of West Nile virus through blood transfusion in the United States in 2002. N Engl J Med 349(13):1236-45. Epub 2003 Sep 18

41. Pecorari M, Longo G, Gennari W, et al (2009) First human case of Usutu virus neuro-invasive infection, Italy, August-September 2009. Euro Surveill 14(50). pii: 19446

42. Platt GS, Way HJ, Bowen ET, et al (1975) Arbovirus infections in Sarawak, October 1968-February 1970 Tembusu and Sindbis virus isolations from mosquitoes. Ann Trop Med Parasitol 69 (1):65-71

43. Rovida F, Percivalle E, Campanini G, et al (2011) Viremic Dengue virus infections in travellers: potential for local outbreak in Northern Italy. J Clin Virol 50(1):76-9. Epub 2010 Nov 4

44. Streit JA, Yang M, Cavanaugh JE, Polgreen PM (2011) Upward trend in dengue incidence among hospitalized patients, United States. Emerg Infect Dis 17(5):914-6

45. Su J, Li S, Hu X, et al (2011) Duck egg-drop syndrome caused by BYD virus, a new Tembusu-related flavivirus. PLoS One 6(3): e18106

46. Tamba M, Bonilauri P, Bellini R, et al (2011) Detection of Usutu virus within a West Nile virus surveillance program in Northern Italy. Vector Borne Zoonotic Dis 11(5):551-7. Epub 2010 Sep 17

47. Tran A, Chastel C (2008) Grossesse et arbovirus transmis par des moustiques : conséquences pathologiques pour la mère et l'enfant. Une revue généarle. Bull Soc Pathol Exot 101(5): 418-23 [http://www.pathexo.fr/documents/articles-bull/T101-53246.pdf]

48. Traore-Lamizana M, Zeller HG, Mondo M, et al (1994) Isolation of West Nile and Bagaza viruses from mosquitoes (Diptera: Culicidae) in central Sénégal (Ferlo). J Med Entomol 31(6):934-8

49. Van Den Hurk AF, Montgomery BL, Northill JA, et al (2006) Short report: the first isolation of Japanese encephalitis virus from mosquitoes collected from mainland Australia. Am J Trop Med Hyg 75(1):21-5

50. Van den Hurk AF, Ritchie SA, Johansen CA, et al (2008) Domestic pigs and Japanese encephalitis virus infection, Australia. Emerg Infect Dis 14(11):1736-8

51. Vázquez A, Ruiz S, Herrero L, et al (2011) West Nile and Usutu viruses in mosquitoes in Spain, 2008-2009. Am J Trop Med Hyg 85(1):178-81

52. Weissenböck H, Kolodziejek J, Url A, et al (2002) Emergence of Usutu virus, an African mosquito-borne flavivirus of the Japanese encephalitis virus group, central Europe. Emerg Infect Dis 8 (7):652-6

53. Weissenböck H, Kolodziejek J, Fragner K, et al (2003) Usutu virus activity in Austria, 2001-2002. Microbes Infect 5 (12):1132-6

54. Wilder-Smith A, Chen LH, Massad E, Wilson ME (2009) Threat of dengue to blood safety in dengue-endemic countries. Emerg Infect Dis 15(1):8-11 